\title{
Evaluation of DWT based image Fusion with three Different Resampling Methods
}

\author{
G. Mamatha ${ }^{1}$, M.V. Lakshmaiah ${ }^{2}$, V. Sumalatha ${ }^{3}$ \\ Assistant Professor, Dept of ECE, JNTUA, Anantapur, India ${ }^{1}$ \\ Assistant Professor, Dept of Physics \& Electronics, SK University, Anantapur, India ${ }^{2}$ \\ Professor, Dept of ECE, JNTUA, Anantapur, India ${ }^{3}$
}

\begin{abstract}
The science of satellite observation is called remote sensing. In optical remote sensing, the multispectral (MS) image which contains color information is produced by three sensors covering the red, green and blue spectral wavelengths. Because of the trade-off imposed by the physical constraint between spatial and spectral resolutions, the MS image has poor spatial resolution. On the contrast, the panchromatic (PAN) images has high spatial resolution but without color information. Image fusion can combine the geometric detail of the PAN image and the color information of the MS image to produce a high-resolution MS image. - Image fusion is powerful tool to include the detail information into single image from many images taken from different sensors at different instants This study is carried out using Cartosat-2 Panchromatic image with $1 \mathrm{~m}$ spatial resolution and Resource sat-1 Liss-IV sensor of 3 bands $b_{2}$, $\mathrm{b}_{3}$ and $\mathrm{b}_{4}$ with $5.8 \mathrm{~m}$ spectral resolution. The images are to be pre-processed to accommodate the fusion algorithm requirements. This pre-processing may include registration and resampling of the MS and PAN images. Image Resampling is the mathematical technique used to alter the scale of an image. Popularly known basic interpolation techniques are nearest neighbor, bilinear and bicubic. These resampled MS and PAN images are fused by using Discrete Wavelet Transformations (DWT) technique. Fusion results are evaluated numerically; and quality metrix are calculated between the fused image and MS image.
\end{abstract}

Keywords: Resampling, DWT, Image Fusion, Image Processing

\section{INTRODUCTION}

Image fusion is a process of combining the relevant information from a set of images into a single image, where the resultant fused image will be more informative and complete than any of the input images. Image fusion techniques can improve the quality and increase the application of these data. Image fusion is extensively used in image and signal processing applications such as medical imaging, aerial and satellite imaging, concealed weapon detection. Remote Sensing systems, particularly those deployed on satellites, provide a repetitive and consistent view of the Earth. The main application of image fusion in remote sensing is merging the greylevel high-resolution panchromatic image and the colored low-resolution multispectral image.

A multi-sensor image fusion [1] scheme overcomes the limitations of a single-sensor image fusion by merging the images from several sensors to form a composite image. The discrete wavelet transform (DWT) is the most commonly used wavelet transform for image fusion. There are some advanced Wavelet families such as contourlet transform, non-subsampled contourlet transform and curvelet transform which have been also used for image fusion. Though their performance is good when compared to discrete wavelet transform, these transforms are computationally cost and require large memory. Hence, the two dimensional Discrete Wavelet Transform (DWT) is becoming one of the standard tools for image fusion [2].

\section{REMote Sensing}

Remote sensing is the science of acquiring information about the Earth's surface without actually being in contact with it. This is done by sensing and recording reflected or emitted energy and processing, analyzing, and applying that information. The first requirement for remote sensing is to have an energy source to illuminate the target. This energy is in the form of electromagnetic radiation. The electromagnetic spectrum ranges from the shorter wavelengths to the longer wavelengths. For most purposes, the ultraviolet or UV portion of the spectrum has the shortest wavelengths which are practical for remote Sensing. Some Earth surface materials, primarily rocks and minerals, fluoresce or emit visible light when illuminated by UV radiation. Electromagnetic energy may be detected either photographically or electronically. An image refers to any pictorial representation, regardless of what wavelengths or remote sensing device has been used to detect and record the electromagnetic energy. Sensors that record electromagnetic energy, electronically record the energy as an array of numbers in digital format. Satellite-based remote sensors are classified by the electromagnetic frequencies they sense and whether they sense these frequencies passively or actively. Among the frequencies of most importance for Earth observations are those in the ultraviolet, visible, infrared and microwave regions of the electromagnetic spectrum.

\section{IMAGE PRE-PROCESSING}

In the image fusion, the starting step is to prepare the input images for fusion process. It is also called as data preprocessing which includes registration [3] and resampling of the input images. Registration [4] is needed to align the corresponding pixels in the input images. This is usually done by geometric normalization of the images. 
If multispectral (MS) and panchromatic (PAN) images are taken from the same sensor they are usually already coregistered and can be directly used for fusion processing. However if the images are taken from different sensors, a registration process is necessary to ensure that pixels in the input images exactly represent the same location on the ground. After registration, resampling of images should be done to make the proportion between the pixel spacing of the PAN and MS images to be same (Spatial domain) or a power of 2 (DWT).

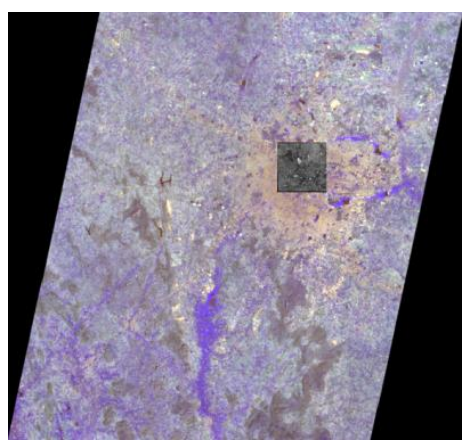

Fig 1. Resampling Image

\section{Image Resampling:}

Image fusion takes place only when the spatial resolution of the input images is same. Image resampling [5] is necessary to get the required spatial resolution of an image. Image Resampling is the mathematical technique used to alter the scale of an image. In image resampling, interpolation and sampling are often combined so that the image is interpolated at only those pixels which need to predict. Popularly known basic interpolation techniques are nearest neighbor, bilinear and bicubic.

Nearest Neighbor: The simplest interpolation from a computational standpoint is the nearest neighbor, where each interpolated output pixel is assigned the value of the nearest sample point in the input image. But it produces blocky result when upsampling is performed and fine result when down sampling is performed.

Bilinear: In this technique new pixels are calculated by using linear interpolation. In upsampling this method operates on $2 \times 2$ cell of pixels surrounding each new pixel location. Here the resampled images are much smoother than nearest neighbor resampled images.

Bicubic: Bicubic resampling method operates on $4 \times 4$ cell of pixels surrounding each new pixel location. This method is recommended for most images and it has a good trade-off between speed and accuracy.

To evaluate the performance of the above said resampling techniques, MS bands viz., Band2 (green), Band3 (red) and Band4 (near infrared) were tested. For the purpose of evaluation, the original size of each image (370x320) is scaled down to a size of $128 \times 128$. Then by using three interpolation techniques, the scaled down images were resampled to the original size images. The original and resampled images are shown in Figures 3-5. After interpolation, the PSNR values between the interpolated images and the standard test images were calculated and represented in Table 1. A higher peak signal to noise ratio would normally indicate the higher quality of the output image. 
$\mathrm{RMSE}=\sqrt{\frac{1}{l * m * n}\left(\sum \sum \sum(F(i, j)-M(i, j))^{2}\right.}$

$l, m, n$ are represent the size of the fused image. $M(i, j)$ is pixel value of MS image and $F(i, j)$ is pixel value of fused image at $(i, j)$ location.

B. Relative Dimensionless Global Error in Synthesis (RDGES):-

It calculates the amount of spectral distortion in the image[33]. It is calculates as

$R D G E S=100 * \frac{h}{l} \sqrt{\frac{1}{N} \sum\left(\frac{R M S E(n)}{\text { mean }(n)}\right)^{2}}$

Where $\mathrm{h}$ is the pixel size of of PAN image and 1 is pixel size of MS image. $\operatorname{RMSE}(n), \operatorname{mean}(n)$ are the root mean square error and mean radiance of individual spectral bands.

C. Relative average spectral error (rase):-

It characterizes the average performance of the method for all bands[32]. It is calculated as

$\operatorname{RASE}=\frac{100}{M} \sqrt{\frac{1}{N} \sum \operatorname{RMSE}(n)^{2}}$

Where $M$ is the mean radiance of the $\mathrm{N}$ spectral bands (B ) of the original MS bands.RMSE(n) is RMSE of the $\mathrm{n}^{\text {th }}$ band of the fused image.

\section{Correlation Coefficient $(C C)$ :-}

It measures the similarity of two images. It ranges from -1 to $+1 .+1$ indicates that two images are highly similar and 1 indicates highly dissimilar. It is calculated as

$C C(F, M)=\frac{\sum(F-\bar{F})(M-\bar{M})}{\left(\sum(F-\bar{F})^{2}\right)\left(\sum(M-\bar{M})^{2}\right)}$

(4)

$F, M$ are represent fused image and multispectral image.

E. $\quad$ Universal Image Quality Index $(Q):-$

It is calculated as

$\mathrm{Q}=\frac{4 \sigma_{f m} \overline{F M}}{\sigma_{f}^{2} \sigma_{m}^{2}\left(\bar{F}^{2}+\bar{M}^{2}\right)}$

Where $\sigma_{f m}$ is covariance between fused image and MS image. $\sigma_{m}^{2}, \sigma_{f}^{2}$ are variance of MS and fused images respectively. $\bar{M}, \bar{F}$ are mean of $\mathrm{MS}$ and fused images respectively.

F. $\quad$ Differences in variances (DIV):-

It is calculated as

DIV $=1-\frac{\text { variance (Fused Image) }}{\text { variance (Orignal Image) }}$

G. Bias:-

Bias measures the error and spectral accuracy[32].

Bias $=1-\frac{\text { mean } \text { (Fused Image })}{\text { mean (Original Image) }}$

Here MS Image is taken as Original Image.

H. $\quad$ Entropy $(H):-$

(6)

(7)

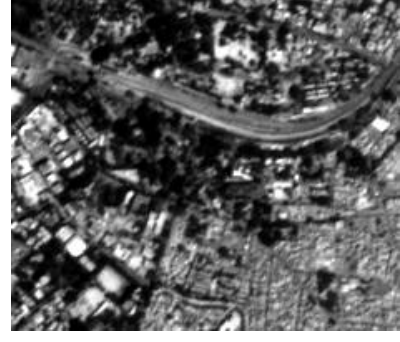

Band3

\section{EXPERIMENTAL RESULTS}

This paper presents fused results of Indian remote sensing satellites images. PAN image size [8] is $1000 \times 1000$ and MS image size is $200 \times 200$. These are taken from National Remote Sensing Centre (NRSC). The results are developed in Earth Resources Data Analysis System (ERDAS) Imagine 2013. The simulated results are as shown in Figure 6.

Image fusion takes place only when the spatial resolution of the input images is same. Image resampling is necessary to get the required spatial resolution of an image. Image Resampling is the mathematical technique used to alter the scale of an image. In image resampling, interpolation and sampling are often combined so that the image is interpolated at only those pixels which need to predict. Popularly known basic interpolation techniques are nearest neighbor, bilinear and bicubic.

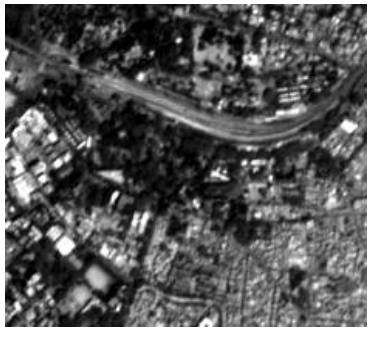

Band2

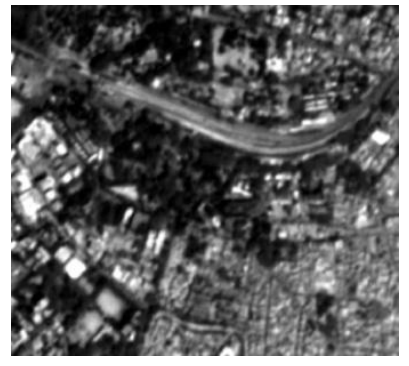

Bilinear

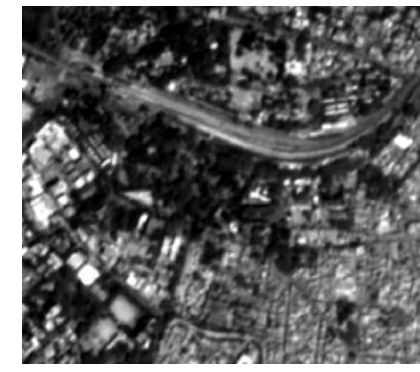

Nearest neighbor

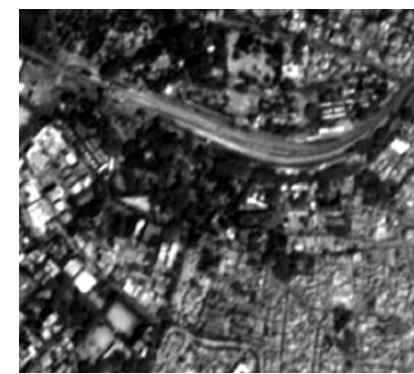

Bicubic
Fig 3. Original and resampled images of Band2

Entropy measures the amount of information contained in the intensity component fused image[3].

Entropy $=-\sum_{i=0}^{L-1} p_{i} \log \left(p_{i}\right)$

Where $p_{i}$ is probability of occurrence of a particular gray level. $\mathrm{L}$ is total number of gray levels.

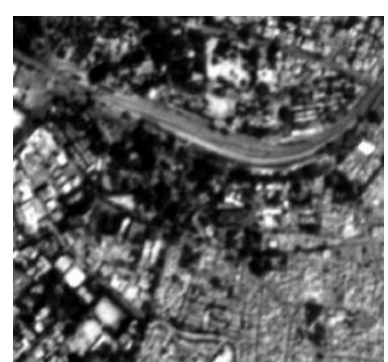

Nearest neighbor 


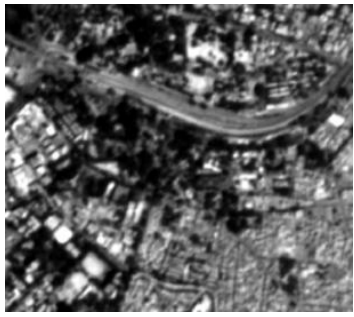

Bilinear

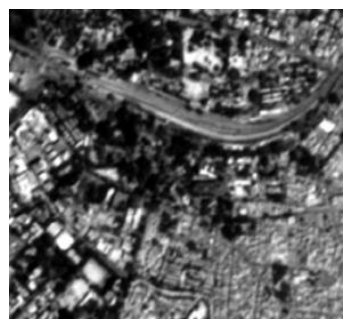

Bicubic

Fig 4. Original and resampled images of Band3

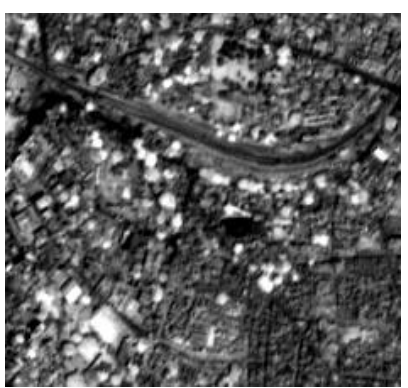

Band4

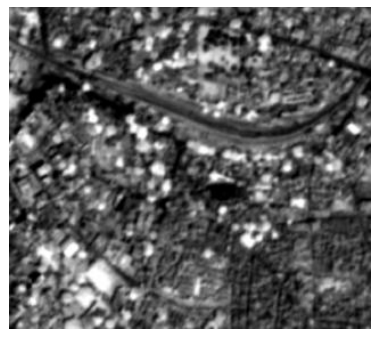

Bilinear

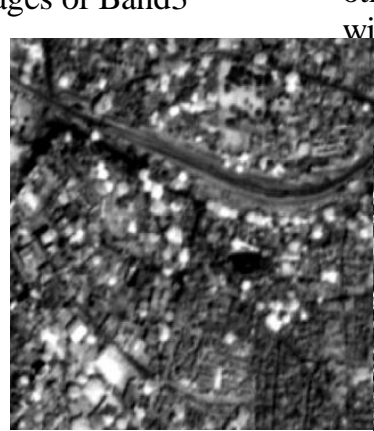

Nearest neighbor

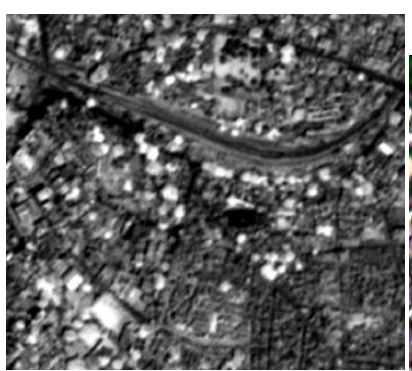

Bicubic

Fig 5. Original and resampled images of Band4

Table 1 PSNR Values of Individual bands

\begin{tabular}{|c|c|c|c|}
\hline $\begin{array}{c}\text { Test } \\
\text { Imagel } \\
\text { Method }\end{array}$ & $\begin{array}{c}\text { Nearest } \\
\text { Neighbor }\end{array}$ & Bilinear & Bicubic \\
\hline Band2 & 22.8916 & 25.5712 & $\mathbf{2 7 . 1 9 4 0}$ \\
\hline Band3 & 28.0435 & 31.3259 & $\mathbf{3 3 . 7 1 0 7 2}$ \\
\hline Band4 & 22.8658 & 22.8658 & $\mathbf{2 7 . 4 2 1 1}$ \\
\hline
\end{tabular}

From the Table 1, it is observed that bicubic interpolation gives a high PSNR values for all Bands as compared to those of bilinear and nearest neighbor interpolation techniques have been used for image fusion.

Table 2. Performance Parameters of Resampled images

\begin{tabular}{|c|c|c|c|}
\hline S.NO. & $\begin{array}{c}\text { NEAREST } \\
\text { NEIGHBOUR } \\
\text { RESAMPLED } \\
\text { WAVELET }\end{array}$ & $\begin{array}{c}\text { BILINEAR } \\
\text { RESAMPLED } \\
\text { WAVELET }\end{array}$ & $\begin{array}{c}\text { BICUBIC } \\
\text { RESAMPLED } \\
\text { WAVELET }\end{array}$ \\
\hline BIAS & 0.003745 & 0.002748 & 0.005646 \\
\hline DIV & 0.047374 & 0.0442536 & 0.040783 \\
\hline CC & 0.850186 & 0.871447 & 0.948242 \\
\hline ENTROPY & 4.874119 & 4.866753 & 4.900492 \\
\hline RDGES & 67.0896 & 60.95322 & 38.9804 \\
\hline Q & 0.849911 & 0.871424 & 0.94798 \\
\hline RASE & 13.15732 & 11.95685 & 7.693807 \\
\hline RMSE & 3.915727 & 3.560047 & 2.308546 \\
\hline
\end{tabular}

From the Table 2, DWT fused method gives better results for bi-cubic resampling technique. The reference values of BIAS, RMSE, RASE are equal to 0. Bi-cubic resampled wavelet image gives better values and close to reference values. The reference values of $\mathrm{CC}, \mathrm{Q}$ are equal to 1 . The entropy ideal value is maximum and RDGES value is minimum. Compare with nearest neighbor and bilinear resampled image $\mathrm{Bi}$-cubic resampling technique provides better results for all parameters. Hence it is recommended for resampling. This paper work will be further extends to other different resampling methods such as Lanczoswindowed sinc kernel operates $4 \times 4,6 \times 6$ and $8 \times 8$ cell of ds. This paper can be further enhanced to a 4-level lets decomposition.

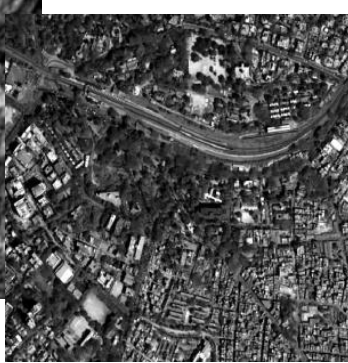

$1 \mathrm{~m}$ Cartosat-2 PAN image

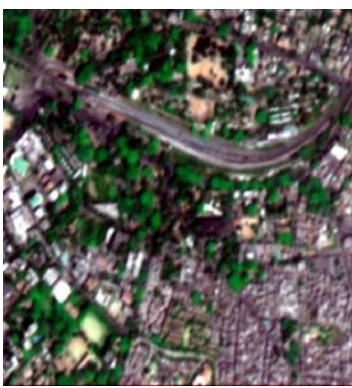

Nearest neighbor MS image

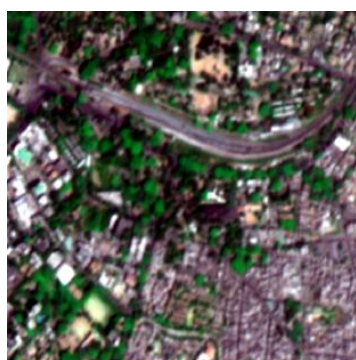

Bilinear resampled image

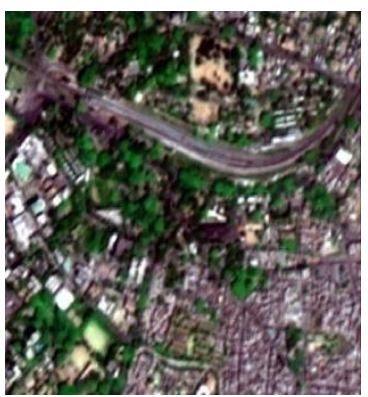

Bi cubic resampled image

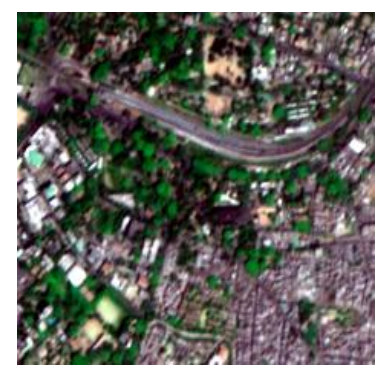

$5 \mathrm{~m}$ resourcesat-1 MS image

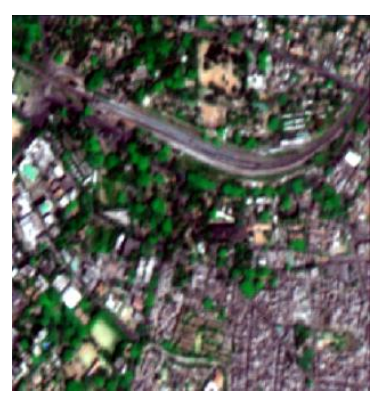

wavelet fused image after nearest neighbor resampling

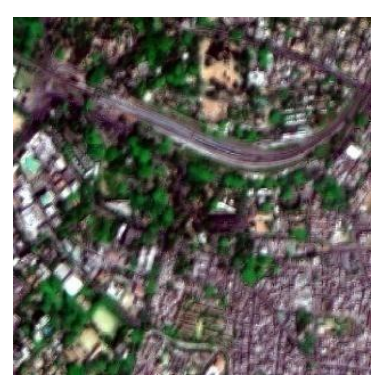

Wavelet fused image after Bilinear resampling

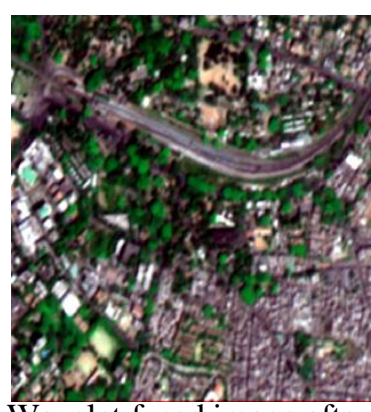

Wavelet fused image after Bicubic Resampling
Fig 6. Wavelet fused Images of each resampled images 


\section{CONCLUSION}

The image fusion methodology developed for different resampling techniques are verified by calculating the PSNR and $\mathrm{CC}$ values. By observing the PSNR \& CC values of different resampling techniques, Bicubic technique results in higher performance as compared to the rest other resampling techniques.

\section{REFERENCES}

[1] C. Pohl and J. L. V. Genderen, "Multisensor image fusion in remote sensing: concepts, methods and applications", Int. J. Remote sensing, vol. 19, no. 5, pp. 823-854,1998.

[2] G. Simone, A. Farina, F.C. Morabito, S.B. Serpico, L. Bruzzone "Image fusion techniques for remote sensing applications". Elsevier.Information Fusion 3 (2002) 3-15.

[3] J. Inglada, V. Muron, D. Pichard, and T. Feuvrier, "Analysis of artifacts in subpixel remote sensing image registration,"IEEE Trans. Geosci. Remote Sens., vol. 45, no. 1, pp. 254-264, Jan. 2007.

[4] L. Brown, "A survey of image registration techniques", ACM Comput.Surv. 24 (4) (1992) 325-376.

[5] Jonathan "Image Resampling," http://www.dlac.com/Resampling.pdf

[6] Gonzalo Pajares,, Jesus Manuel de la Cruz "A Wavelet Based Image Fusion tutorial'. Journal on Pattern Recognition.Elsevier. 2004.

[7] Q. Du, O. Gungor, and J. Shan, "Performance evaluation for pansharpening techniques," inProc. IEEE Int. Geosci. Remote Sens. Symp., pp. 4264-4266, 2005.

[8] Peter Bunting,FrédéricLabrosse, Richard Lucas "A multi-resolution area-based technique for automatic multi-modalimage registration. Elsevier.Image and Vision Computing 28 (2010) 1203-1219 